\title{
A Finite Element Study of the Relationship Between Bone Fibril Elasticity and Degree of Mineralization
}

\author{
Franklin Ogidi \\ Biosystems Engineering \\ University of Manitoba \\ Winnipeg, Canada \\ ogidif@myumanitoba.ca
}

\author{
Qiang Zhang \\ Biosystems Engineering \\ University of Manitoba \\ Winnipeg, Canada \\ qiang.zhang@umanitoba.ca
}

\author{
Yunhua Luo \\ Mechanical Engineering \\ University of Manitoba \\ Winnipeg, Canada \\ yunhua.luo@umanitoba.ca
}

\begin{abstract}
This paper presents a two-dimensional (2D) finite element model of the mineralized collagen fibril. The model was developed to investigate the relationship between fibril elasticity and degree of mineralization, the latter is measured by volume fraction of minerals. Mineralized fibrils were modeled as two-phase composite materials with mineral platelet inclusions embedded in the collagen matrix. Fibril elasticity moduli were determined by finite element analyses. It was found that the fibril elastic modulus increases slowly with the volume fraction of minerals up to a volume fraction of $39.2 \%$. Beyond this point, the elastic modulus increases rapidly with volume fraction. This rapid increase is probably attributed to the sharp gain in von Mises strain within the fibril resulting from a decrease in axial spacing between hydroxyapatite crystals. These results provide insights into the mechanical properties of bone at the nano-mesoscale. The results from the finite element modelling are compared with predictions from theoretical models such as the Mori-Tanaka Scheme, the Self-consistent scheme, and the Voigt-Reuss bounds. Whereas there are considerable differences between theoretical predictions and finite element results, similar trends can still be observed, indicating that finite element modelling is a promising approach to understand the effects of bone chemical composition on its mechanical behaviour.

Index Terms-bone; mineralized collagen fibril; hydroxyapatite; finite element method; elasticity; volume fraction
\end{abstract}

\section{INTRODUCTION}

Bone is a rigid material that serves, among a variety of functions, as a light-weight frame to support other tissues in the body. The mechanical functions of bone are achieved through its complex hierarchical structure (Fig. 1), with mechanical properties depending on its architecture at all structural levels $[1,2]$. The focus of this paper is the mineralized collagen fibril (MCF), which is the main component of bone at the lowest structural level [3].

The MCF can be considered as a composite material consisting of organic matrix reinforced by inorganic minerals [4]. Cross-linked type I collagen (or tropocollagen) molecules make up about $90 \%$ weight of the proteins in the organic matrix while non-collagenous proteins comprise the other $10 \%$ [5]. The tropocollagen molecules are about $300 \mathrm{~nm}$ long and are staggered with a $67 \mathrm{~nm}$ period in the axial direction [1]. The staggering produces overlap and gap regions, which have been estimated to take up about $40 \%$ and $60 \%$ of the axial period, respectively [3]. The inorganic phase, which is mainly composed of hydroxyapatite (HA) crystals start to nucleate in the gap region [6] and grow predominantly in the longitudinal direction of the fibril [1]. Although reported experimental results vary in terms of the geometric properties of HA crystals, most researchers agree that the minerals are plateletlike structures that are 15-200 $\mathrm{nm}$ long, 10-80 $\mathrm{nm}$ wide and 2-7 $\mathrm{nm}$ thick [4, 7]. While the HA crystals are stiff and brittle, contributing to the strength and stiffness of bone, the collagen matrix is tough and ductile, which provides bone with a high capacity for energy dissipation during mechanical deformation [9]. Together, these major constituents of the MCF combine to achieve a compromise such that the composite is both stiff and tough [7].

The extent to which the overall elasticity of the collagen fibril is affected by the combination of the mechanical properties of its individual constituents is largely determined by the volume fraction of minerals in the fibril [8]. Indeed, a parametric study of the MCF conducted by [2] has shown that the volume fraction of minerals has the most influential effect on the elastic properties of the MCF. Furthermore, the volume fraction of minerals can have far-reaching effects at the organ level as bone diseases such as osteomalacia and osteogenesis imperfecta arise because of abnormal degree of mineralization within the bone [8, 10]. Therefore, it is important to characterize the relationship between the volume fraction of minerals and the elastic properties of collagen fibrils.

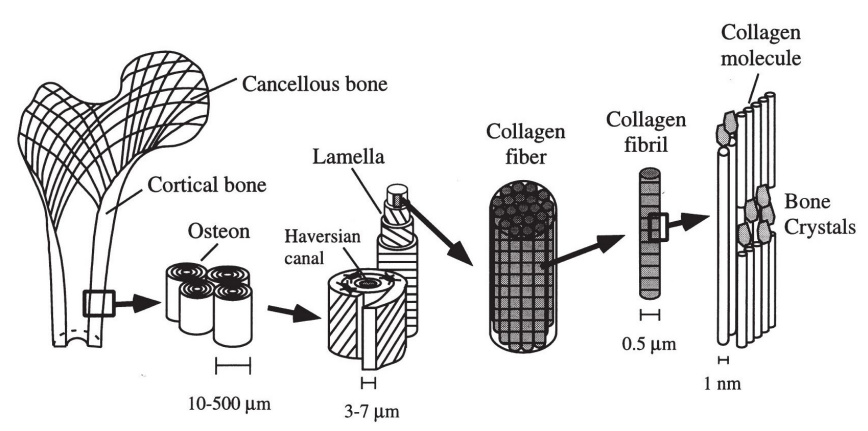

Fig. 1. The hierarchical structure of bone, from the macrostructure on the far left to the sub-nanostructure on the far right [1] 
To estimate the elastic properties of the MCF, most previous studies have employed analytical, numerical, or computational methods. Analytical methods involve the use of mathematical formulations, based on either the strength of materials or micromechanics theories, and model the properties of bone at the level of individual constituents. The MoriTanaka (MT) scheme, for instance, was developed [11] for calculating the average internal stress in a matrix containing homogenous inclusions. The MT theory was later reformulated [12] for two-phase composite materials containing aligned or randomly oriented ellipsoidal inclusions. In addition to the matrix-inclusion interactions, the MT scheme also accounts for inclusion-inclusion interactions [4]. Similarly, the SelfConsistent scheme (SCS), which was originally developed in [13] and [14], predicts the elastic properties of crystalline aggregates with randomly oriented grains and no matrix. Like the MT scheme, the SCS was reformulated in [15] and [16] for predicting the elastic moduli of two-phase composite materials, where the phases are required to be a homogenous matrix and ellipsoidal or spherical inclusions. Although these models are formulated in terms of the volume fraction of minerals, they are typically based on simplified assumptions and cannot accurately account for the geometrical arrangement of the collagen and HA crystals relative to each other [4].

In recent years, numerical and computational methods like the Finite Element Method (FEM) have found application in the analysis of the MCF [2,9]. The FEM can more accurately account for the geometric arrangement of individual constituents of the MCF [4]. For instance, Yuan et al [2] utilized the FEM to show the effects of HA crystal thickness, length and spacing on the apparent modulus of the collagen and mineral phases. Recently, techniques like computational electrodynamics [17] and molecular dynamics simulations [18] have been applied in the study of the MCF. However, these approaches are beyond the scope of this paper.

In light of the preceding discussion, this paper will investigate the relationship between the volume fraction of minerals and the elastic modulus of the MCF by using the FEM to analyze a two-dimensional (2D) model of the collagen fibril. A differentiating element of this study is the modelling of the HA crystals as inclusions in the collagen matrix (like the MT scheme and the SCS but accounting for the geometric arrangement of the individual constituents), as opposed to longitudinally growing platelets [19]. By comparing the FEM results to the results of micromechanics-based analytical models, this study aims to deepen the understanding of the behaviour of bone at the nano-mesoscale.

\section{Methodology}

\section{A. Assumptions and Fibril Finite Element Model}

Fig. 2 shows the two-dimensional (2D) model of the mineralized collagen fibril (MCF) that was created for this study. The 2D structure has been shown to provide a sufficient understanding of the elastic properties of bone at the nanoscale, even with its simplicity compared to a three-dimensional model [2].
The model considers the MCF as a two-phase composite consisting of a homogenous collagen matrix and HA crystals. This assumption is made for simplicity. It is also a reasonable assumption to make, as the collagen matrix makes up about $90 \%$ of the bone organic content and the mineral crystals make up around $90 \%$ of the bone inorganic content [10]. As summarized in Table I, the length of the overall structure of the model is $300 \mathrm{~nm}$, representing the length of a tropocollagen molecule (Fig. 2), whereas the width of the model was set to $100 \mathrm{~nm}$. The collagen matrix is modelled as a homogenous matrix and the effects cross-linking are not considered. On the other hand, the HA crystals are modelled as fixed-sized platelet inclusions in the collagen matrix, as opposed to lengthwisegrowing minerals as in [19]. The dimensions of the minerals were fixed at $40 \mathrm{~nm} \times 3 \mathrm{~nm}$, which falls within the range of reported values in the literature $[4,7]$. Although recent studies have shown evidence of HA crystals both inside and outside of the collagen fibril [17, 21, 26], extrafibrillar mineral crystals are not considered in this study.

In the 2D model, isotropic properties are assumed for the collagen matrix and the HA crystals. As outlined in Table I, a Young's modulus of $114 \mathrm{GPa}$ and a Poisson's ratio of 0.23 were used for the HA crystals [21]. These values were experimentally derived for a single crystal [22]. Also following [21], the Young's modulus and Poisson's ratio of the collagen matrix were set to $2.4 \mathrm{GPa}$ and 0.28 , respectively.

In terms of geometrical arrangement, a staggered pattern of arrangement of the HA crystals has been shown to be mechanically superior compared to a strictly parallel arrangement [3]. As such, the staggered arrangement is employed in the model

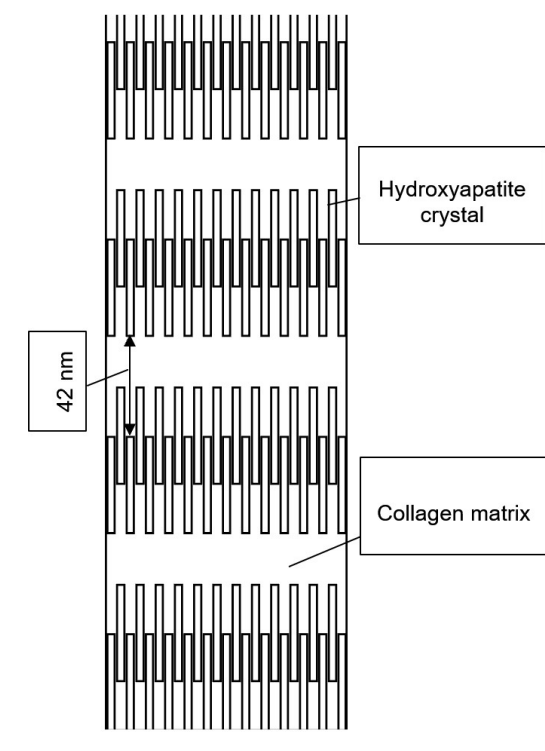

a)

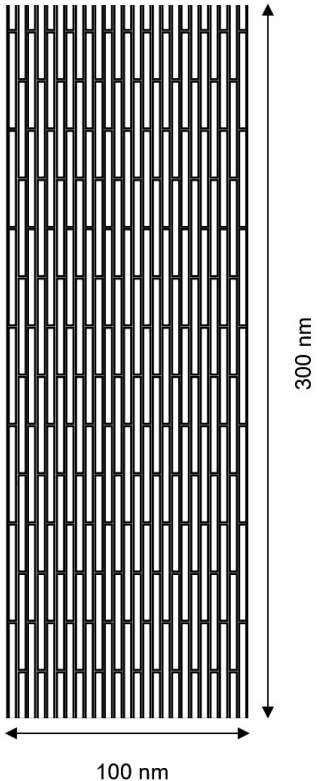

b)
Fig. 2. A representative model of the mineralized collagen fibril consisting of hydroxyapatite platelet inclusions in a collagen matrix. a) shows the model at a mineral volume fraction of $39.2 \%$ while b) shows the mineralized model at $73.3 \%$ volume fraction of minerals. 
TABLE I

GEOMETRIC AND MATERIAL PROPERTIES OF CONSTITUENTS OF MINERALIZED COLLAGEN FIBRIL [21], WHERE E IS THE YOUNG'S MODULUS AND $\nu$ IS THE POISSON'S RATIO.

\begin{tabular}{|c|c|c|c|c|}
\hline Material & Length $(\mathbf{n m})$ & Width $(\boldsymbol{n m})$ & $\boldsymbol{E}$ & $\nu$ \\
\hline Collagen Matrix & 300 & 100 & 2.4 & 0.28 \\
\hline HA Crystals & 40 & 3 & 114 & 0.23 \\
\hline
\end{tabular}

presented in this paper (Fig. 2). However, for this study, the minerals are not staggered with the typical $67 \mathrm{~nm}$ period. This is done to expand the range of volume fractions that the model can simulate beyond the limits posed by the natural arrangement of the minerals in the MCF. A combination of the fixedsize platelets and the staggered arrangement pattern results in a $73.3 \%$ volume fraction of minerals at full mineralization (Fig. 2b). Jäger et al [3] proposed a 56\% limit to the volume fraction of minerals for a model of the collagen fibril with a staggered arrangement of minerals incorporating the periodic boundary condition. Since the MCF is a "universal" structure found in tendons, enamel, and dentin, simulating a wider range of volume fractions may give insights into the behaviour of those materials.

\section{B. Finite Element Implementation}

The model in Fig. 2 was constructed and analyzed in ANSYS Mechanical APDL 18.1. Both the collagen matrix and the mineral crystals were modelled as plane stress/strain elements with up to 8 nodes and 2 degrees of freedom at each node. To simulate the variation of volume fractions of minerals in the model, the fix-sized HA crystals were systematically added as inclusions to the collagen matrix using the overlap Boolean operation. This operation sums multiple entities to create new entities that include all the parts of the original but maintains the boundaries at the overlap zones [20]. Compared to lengthwise-growing platelets, modelling the mineral crystals as inclusions eliminates the length of the minerals as a potential confounding factor in the results.

To simulate tensile testing, the model was fixed at the bottom edge while displacement boundary conditions were applied to the top edge. The displacement boundary conditions were applied in 10 load steps for displacements ranging from $0.1 \mathrm{~nm}$ to $1 \mathrm{~nm}$, with a $0.1 \mathrm{~nm}$ increment at each step. For each volume fraction, the Young's modulus of the MCF is determined from the slope of the corresponding stress-strain curve. Prior to the mechanical testing, a mesh convergence study was conducted to minimize the effects of mesh size on the accuracy of the results. The reaction force at the bottom surface was the variable of interest in the convergence study.

The results of the FEM simulations were compared with the Mori-Tanaka (MT) scheme and the Self-consistent scheme (SCS). These analytical methods were chosen because they are closely related to the inclusion approach used in the finite element analysis. The calculations for the MT scheme and the SCS were performed using MMTensor, an open-source collection of MATLAB scripts for performing micromechanical computations [23]. The Voigt and Reuss models were also included in the comparison to represent the upper and lower bounds of the elastic modulus of the composite material. The Voigt-Reuss bounds are based on strength of materials and are formulated for analyzing multi-phase composites, assuming uniform strain and uniform stress in the material [4].

\section{RESUlTS AND Discussion}

In this section, the link between collagen fibril elasticity and the volume fraction of minerals, resulting from the finite element analysis, is presented. This relationship is explained considering the methodology used to obtain the results and compared to previous research. Possible limitations of the study and recommendations for future research are also outlined.

\section{A. Convergence Study}

To minimize the effects of mesh size on the accuracy of the results, the element edge size of the fully mineralized model was refined until the solution to the reaction force converged. As shown in Table II, convergence is reached at the point where a decrease in the element edge size results in a negligible change in the reaction force. A smaller element edge size means that the model is being approximated by more elements and nodes, increasing the accuracy of the solution. However, the higher the number of elements used to approximate the model, the more time it takes to discretize the model and find a solution. Therefore, to maintain the balance between computational time and the accuracy of the solution, an element edge size of 0.5 was selected for the FEM simulations.

\section{B. Finite Element Modelling Results}

The 2D model of the collagen fibril was analyzed under tensile loading in the longitudinal direction, while the volume fraction of minerals varied from $0 \%$ to $73.3 \%$. Fig. 3 shows the observed relationship between the fibril elasticity and the volume fraction of minerals resulting from the FEM simulations compared with other analytical solutions. The elastic modulus of the MCF increases monotonically as the volume fraction of mineral increases. The results show a gradual increase in

TABLE II

RESULTS OF THE MESH CONVERGENCE STUDY WITH THE CHOSEN ELEMENT EDGE LENGTH HIGHLIGHTED IN BOLD.

\begin{tabular}{|c|c|c|c|}
\hline $\begin{array}{c}\text { Element Edge } \\
\text { Length }\end{array}$ & $\begin{array}{c}\text { Number of } \\
\text { Elements }\end{array}$ & $\begin{array}{c}\text { Number of } \\
\text { Nodes }\end{array}$ & $\begin{array}{c}\text { Reaction Force } \\
(\boldsymbol{N})\end{array}$ \\
\hline 5 & 3579 & 10554 & $1.2750 \mathrm{E}-09$ \\
\hline 3 & 5627 & 17136 & $1.2575 \mathrm{E}-09$ \\
\hline 2 & 11704 & 35557 & $1.2508 \mathrm{E}-09$ \\
\hline 1 & 30413 & 91912 & $1.2464 \mathrm{E}-09$ \\
\hline 0.9 & 42684 & 128967 & $1.2440 \mathrm{E}-09$ \\
\hline 0.8 & 49160 & 148505 & $1.2443 \mathrm{E}-09$ \\
\hline 0.7 & 69508 & 209715 & $1.2443 \mathrm{E}-09$ \\
\hline 0.6 & 89586 & 270107 & $1.2424 \mathrm{E}-09$ \\
\hline $\mathbf{0 . 5}$ & $\mathbf{1 2 2 2 2 5}$ & $\mathbf{3 6 8 2 5 6}$ & $\mathbf{1 . 2 4 1 2 E - 0 9}$ \\
\hline 0.4 & 206175 & 620564 & $1.2405 \mathrm{E}-09$ \\
\hline
\end{tabular}




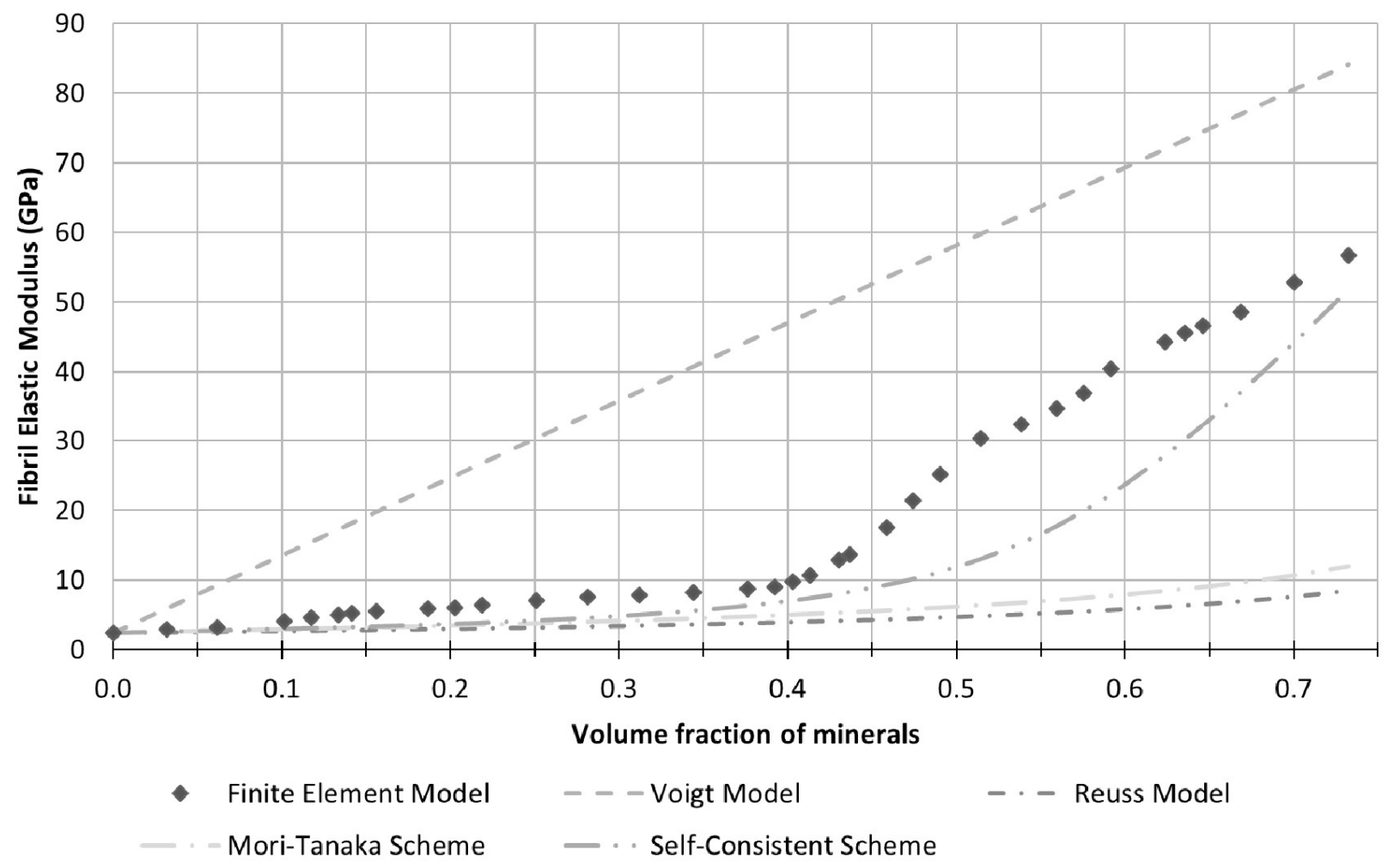

Fig. 3. Comparison of the relationship between mineralized collagen fibril elastic modulus and volume fraction of minerals predicted by the finite element method simulations and other analytical models. For all models shown, the elastic modulus of the collagen matrix is 2.4 GPa, while the elastic modulus of the hydroxyapatite crystals is $114 \mathrm{GPa}$

fibril elastic modulus between $0 \%$ and $39.2 \%$ volume fraction of minerals, followed by a sharp gain in elasticity. In fact, the volume fraction of $39.2 \%$ is important because, beyond that point, the axial distance between additional platelet inclusions and existing HA crystals decreases from $42 \mathrm{~nm}$ to $1 \mathrm{~nm}$.

A closer look at the strain distribution in the model reveals why the fibril elastic modulus increased so rapidly as the axial spacing between the HA crystal inclusions shrunk. For instance, the von Mises strain distribution in the model at a volume fraction of $39.2 \%$ shows that the large axial spacing between the minerals allows the strain to be dissipated through the collagen matrix, which reduces the stress on HA crystals (Fig. 4). After the inclusion of four HA crystals, increasing the volume fraction of minerals to $40.3 \%$, strain concentration regions can be observed in the $1 \mathrm{~nm}$ spacing near the newly added mineral crystals (Fig. 5). These strain concentration regions increase the magnitude of the strain transferred between the HA crystals. Since the minerals are stiffer than the collagen matrix, the large strain transfer increases the stress in the mineral phase. This, in turn, increases the elastic modulus of the composite MCF.

The observations from the strain and stress distribution in the finite element model suggest that the decrease in the axial spacing between the mineral crystals has a significant effect on the fibril elastic modulus. This is also applicable in the model of the MCF with longitudinally growing minerals - as the HA crystals grow, the axial spacing between the mineral phase decreases, leading to an increase in both the volume fraction of minerals and the fibril elastic modulus. However, due to the periodic boundary condition in collagen fibril, there may be a limit to how close minerals can get in the longitudinal direction. Nevertheless, the FEM simulation results provide a deeper understanding of the possibly abnormal case where HA crystals grow beyond the gap regions.

\section{Comparisons}

The comparison between theoretical predictions and FEM simulations is illustrated in Fig. 3. The Voigt and Reuss models serve as a sanity check, as they represent the theoretical upper and lower bounds for the elastic modulus of composite materials, respectively. The FEM results fall well within these bounds, suggesting that the results are reasonable, at least from a strength of materials perspective.

The Mori-Tanaka (MT) scheme and the Self-Consistent scheme (SCS) both model matrix-inclusion interactions, like the approach used in this study. Whereas the predictions from both the MT scheme and the SCS diverge significantly from those of the finite element analysis, especially at larger volume fractions of minerals, a more similar trend can be observed between the results of the SCS and the FEM simulations. The 

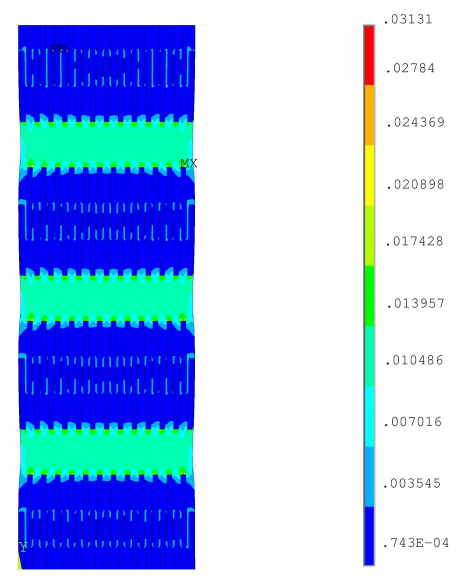

Fig. 4. Von Mises elastic strain distribution in the model at a volume fraction of $39.2 \%$ and a tensile deformation of $1 \mathrm{~nm}$.

differences between the results of the FEM and the inclusionbased analytical models may be attributed to the differences in the shape of the inclusions - while the HA crystals are modelled as rectangular platelet inclusions in the finite element model, they are considered as ellipsoidal/spherical inclusions in the MT scheme and the SCS. These differences are further accentuated by the fact that the analytical models cannot accurately account for the geometrical arrangement of the constituents with respect to each other [4]. Nonetheless, the similarities in the trends of the predicted results show the promise of using the FEM approach.

\section{Limitations}

To keep the model simple, constituents of the MCF such as non-collagenous proteins and water were excluded. Although these excluded components are not found in substantial amounts in the MCF compared to the constituents considered in this study, their interactions with the predominant constituents may play a role in modulating the overall elasticity of the MCF. For instance, Nyman et al [24] reported a decrease in bone strength at a water loss above $9 \%$. The researchers speculate that water loss in the collagen matrix affects the toughness of bone, while water loss in the HA crystals affects the strength of bone. Similarly, recent experimental evidence has shown that the HA crystals have amorphous domains, which reduce the average modulus [25]. Consequently, the investigation of fluid-solid interaction and non-linear material properties are likely to yield more realistic results even though they may lead to more complex analyses.

The model presented in this paper also excludes extrafibrillar minerals, which, as recent evidence suggests, may be a crucial element of the MCF. For instance, using the FEM, Abueidda et al [9] found that a model of the MCF with mineral crystals on the outside of the collagen phase produced results that are closer to results from micropillar mechanical tests, compared to a model where the minerals are inside the
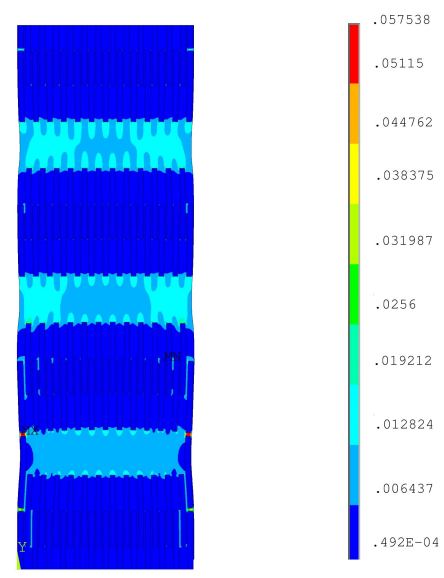

Fig. 5. Von Mises elastic strain distribution in the model at a volume fraction of $40.3 \%$ and a tensile deformation of $1 \mathrm{~nm}$.

collagen phase. Other recent studies show that it is more likely that both intrafibrillar and extrafibrillar mineral crystals act together to determine the mechanical properties of bone [17, $21,26]$. Future research should look further into the role of extrafibrillar minerals in determining bone strength, perhaps using an axisymmetric model instead of a plane stress model to simplify the three-dimensional structure of the collagen fibril.

\section{CONCLUSiOnS}

This study used the FEM to characterize the relationship between the elastic modulus of the mineralized collagen fibril and the volume fraction of minerals. The FEM simulations involved varying the volume fraction of minerals from $0 \%$ to $73.3 \%$ in a $2 \mathrm{D}$ model of the mineralized collagen fibril consisting of a collagen matrix and hydroxyapatite platelet inclusions under tensile loading. The results show a monotonic relationship between the fibril elastic modulus and the volume fraction of minerals and provide insights into the effects of the axial distance between the mineral crystals on the fibril elasticity.

\section{REFERENCES}

[1] J. Y. Rho, L. Kuhn-Spearing, and P. Zioupos, "Mechanical properties and the hierarchical structure of bone", Medical Engineering Physics, 20(2), pp. 92-102, 1998.

[2] F. Yuan, S. R. Stock, D. R. Haeffner, J. D. Almer, D. C. Dunand, and L. C. Brinson, "A new model to simulate the elastic properties of mineralized collagen fibril", Biomechanics and Modeling in Mechanobiology, 10(2), pp. 147-160, 2011

[3] I. Jäger, and P. Fratzl, "Mineralized collagen fibrils: a mechanical model with a staggered arrangement of mineral particles", Biophysical Journal, 79(4), pp. 1737-1746, 2000.

[4] E. Hamed, and I. Jasiuk, "Elastic modelling of bone at nanostructural level", Materials Science and Engineering: R: Reports, 73(3-4), pp. 2749, 2012.

[5] J. D. Currey, Bones: structure and mechanics, Princeton University press, 2013.

[6] S. Weiner, and W. Traub, "Organization of hydroxyapatite crystals within collagen fibrils", Federation of European Biochemical Societies Letters, 206(2), pp. 262-266, 1986. 
[7] P. Fratzl, H. S. Gupta, E. P. Paschalis, and P. Roschger, "Structure and mechanical quality of the collagen-mineral nano-composite in bone", Journal of Materials Chemistry, 14(14), pp. 2115-2123, 2004.

[8] Y. Bala, D. Farlay, and G. Boivin, "Bone mineralization: from tissue to crystal in normal and pathological contexts", Osteoporosis International, 24(8), pp. 2153-2166, 2013.

[9] D. W Abueidda, F. A. Sabet, and I. M. Jasiuk, "Modelling of stiffness and strength of bone at nanoscale", Journal of Biomechanical Engineering, 139(5), 051006, 2017.

[10] Y. Luo, Image-based multilevel biomechanical modeling for fall-induced hip fracture. Springer International Publishing, 2017.

[11] T. Mori, and K. Tanaka, "Average stress in matrix and average elastic energy of materials with misfitting inclusions", Acta Metallurgica, 21(5), pp. 571-574, 1973.

[12] Y. Benveniste, "A new approach to the application of Mori-Tanaka's theory in composite materials", Mechanics of Materials, 6(2), pp. 147$157,1987$.

[13] A. V. Hershey, "The elasticity of an isotropic aggregate of anisotropic cubic crystals", Journal of Applied mechanics-transactions of the ASME, 21(3), pp. 236-240, 1954.

[14] E. Kröner, "Calculation of the elastic constant of the multi-crystal from the constants of the single crystal", ["Berechnung der elastischen Konstanten des Vielkristalls aus den Konstanten des Einkristalls" Zeitschrift für Physik, 151(4), pp. 504-518, 1958].

[15] R. Hill, "A self-consistent mechanics of composite materials", Journal of the Mechanics and Physics of Solids, 13(4), pp. 213-222, 1965.

[16] B. Budiansky, "On the elastic moduli of some heterogeneous materials", Journal of the Mechanics and Physics of Solids, 13(4), pp. 223-227, 1965.

[17] A. Kurfürst, P. Henits, C. Morin, T. Abdalrahman, and C. Hellmich, "Bone ultrastructure as composite of aligned mineralised collagen fibrils embedded into a porous polycrystalline matrix: confirmation by computational electrodynamics", Frontiers in Physics, 6, 125, 2018.

[18] Z. Qin, A. Gautieri, A. K. Nair, H. Inbar, and M. J. Buehler, "Thickness of hydroxyapatite nanocrystal controls mechanical properties of the collagen-hydroxyapatite interface", Langmuir, 28(4), pp. 1982-1992, 2012.

[19] A. Vercher, E. Giner, C. Arango, J. E. Tarancón, and F. J. Fuenmayor, "Homogenized stiffness matrices for mineralized collagen fibrils and lamellar bone using unit cell finite element models", Biomechanics and modeling in mechanobiology, 13(2), pp. 437-449, 2014.

[20] ANSYS® Academic Research Mechanical, Release 18.1, Help System, "Sculpting Your Model with Boolean Operations", ANSYS, Inc.

[21] S. Nikolov, and D. Raabe, "Hierarchical modeling of the elastic properties of bone at submicron scales: the role of extrafibrillar mineralization", Biophysical Journal, 94(11), pp. 4220-4232, 2008.

[22] R. S. Gilmore, and J. L Katz, "Elastic properties of apatites", Journal of Materials Science, 17(4), pp. 1131-1141, 1982.

[23] Martin Moesen, MMTensor 1.0. File Exchange - MATLAB Central, 2011. https://www.mathworks.com/matlabcentral/fileexchange/32891 $(2019 / 03 / 06)$.

[24] J. S. Nyman, A. Roy, X. Shen, R. L. Acuna, J. H. Tyler, and X. Wang, "The influence of water removal on the strength and toughness of cortical bone", Journal of Biomechanics, 39(5), pp. 931-938, 2006.

[25] C. F. Koch, S. Johnson, D. Kumar, M. Jelinek, D. B. Chrisey, A. Doraiswamy, C. Jin, R. J. Narayan, and I. N. Mihailescu, "Pulsed laser deposition of hydroxyapatite thin films", Materials Science and Engineering: C, 27(3), pp. 484-494, 2007.

[26] A. K., Nair, A. Gautieri, and M. J. Buehler. "Role of intrafibrillar collagen mineralization in defining the compressive properties of nascent bone", Biomacromolecules, 15(7), pp.2494-2500, 2014 